\title{
CRESCIMENTO E CARACTERÍSTICAS ANATÔMICAS DE CAFEEIRO (Coffea arabica L.) E SERINGUEIRA (Hevea brasiliensis Muell. Arg.) EM DIFERENTES SISTEMAS DE CULTIVO: MONOCULTIVO E CONSÓRCIO ${ }^{1}$
}

\author{
Growth and anatomical characteristics of coffee (Coffea arabica $\mathbf{L}$.) and rubber \\ (Hevea brasiliensis Muell. Arg.) trees cultivated in single and intercropped systems
}

\author{
Cláudio Roberto Meira de Oliveira ${ }^{2}$, Angela Maria Soares ${ }^{3}$, \\ Luiz Edson Mota de Oliveira ${ }^{4}$, Evaristo Mauro de Castro ${ }^{5}$, João Paulo R. Alves Delfino Barbosa ${ }^{6}$
}

RESUMO

Objetivou-se avaliar neste trabalho as influências do sistema de cultivo em plantas de seringueiras (Hevea brasiliensis Muell Arg.) e de cafeeiros (Coffea arabica L.) na fase de estabelecimento do plantio. Foram avaliadas características de crescimento das plantas de seringueira e a estrutura foliar de cafeeiros e seringueiras em condições de monocultivo e dois tipos de consórcio: renque e margem. Pela análise dos resultados obtidos evidenciou-se que o sistema de cultivo não influenciou o padrão de crescimento das plantas de seringueira. Em geral, o sistema de cultivo não influenciou a anatomia foliar dos cafeeiros nessa fase do cultivo.

Termos para indexação: Consórcio, café, seringueira, crescimento, anatomia foliar.

\section{ABSTRACT}

This study aimed to evaluate the influence of the cropping system in anatomical characteristics and growth of the rubber tree (Hevea brasiliensis Muell. Arg.) and coffee (Coffea arabica L.), in the beginning of the cultivation period. It was evaluated the growth characteristics of rubber tree plants and the leaf structure in both species in single and intercropped cultivation. The results showed that the cropping system did not influence the rubber tree. In general, the cropping system did not influence the coffee leaf structure, in that developmental phase of cultivation.

Index terms: Intercropping, coffee, rubber, growth, leaf structure.

(Recebido para publicação em 23 de abril de 2002 e aprovado em 23 de julho de 2002)

\section{INTRODUÇÃO}

O plantio consorciado do café com espécies arbóreas vem sendo utilizado em várias regiões produtoras de café, por apresentar vantagens sócio-econômicas e biológicas em relação ao cultivo tradicional em monocultivo (SANTOS et al., 2000). Embora esse sistema seja considerado menos produtivo por vários pesquisadores e produtores, deve-se levar em conta que os cafezais em condições de pleno sol requerem tratos culturais mais intensivos e uma densidade maior de plantio (PEETERS et al., 2003).

O consórcio cafeeiro-seringueira tem sido estudado, recomendado e utilizado com vantagens para ambas as culturas em algumas regiões produtoras de café e borracha no Brasil (FANCELLI, 1986, 1990; PEREIRA, 1992; PEREIRA et al., 1994; VENEZIANO et al., 1994; PEREIRA et al., 1998) e em outros países como Java (DIJKMAN, 1951; IRC, 1992). Esse sistema surge como uma alternativa altamente promissora, pois permite a diversificação da produção, proporcionando estabilidade econômica ao agricultor, e ainda contribui para suprir o déficit de borracha natural no País (PEREIRA et al., 2000). O plantio de cafeeiros com espécies arbóreas, entretanto, pode propiciar uma competição por luz, água e nutrientes, que podem refletir em uma série de alterações no comportamento fisiológico das plantas consorciadas, causando não só alterações na

\footnotetext{
1. Parte da dissertação do primeiro autor apresentada àUnivrsidade Federal de Lavras/UFLA - Caixa Postal 37 - 37200-000 - Lavras, MG, como parte das exigências para obtenção do grau de "Mestre" em Fisiologia Vegetal.

2. MSc., Engenheiro Agrônomo - Pós-graduando do Departamento de Biologia da UFLA.

3. Professora Adjunta do Departamento de Biologia da UFLA.

4. Professor Titular do Departamento de Biologia da UFLA.

5. Professor Adjunto do Departamento de Biologia da UFLA.

6. Estudante de Engenharia Agronômica - Bolsista PIBIC-CNPq/UFLA.

Financiado pela EMBRAPA/PNP\&D-CAFÉ.
} 
sua produção e crescimento, como também mudanças de caráter mais específico, como alterações morfológicas e anatômicas (PEETERS, 2003; SCHALLER, 2003).

A estrutura foliar pode ser um forte indicador da disponibilidade de luz durante as fases de crescimento das plantas. O aumento dos níveis de luz proporciona aumentos na espessura foliar, massa foliar, epiderme, parênquima e número total de células das folhas (ESAU, 1977; ABRAMS e MOSTOLLER, 1995; CASTRO et al., 1998; LEE et al., 2000). Essas modificações ocorrem pela variação das divisões celulares, padrão de expansão celular e conseqüente modificação na espessura foliar (FAILS et al., 1982). Diversos estudos demonstram também que o incremento na intensidade luminosa aumenta a freqüência estomática por unidade de área foliar (ASHTON e BERLYN, 1992; CASTRO et al., 1998).

Este trabalho foi realizado com o objetivo de avaliar a influência do sistema de cultivo no crescimento e na anatomia foliar de cafeeiros e seringueiras em consórcio e monocultivo, na fase de estabelecimento dos sistemas de plantio estudados.

\section{MATERIAL E MÉTODOS}

O experimento foi conduzido de outubro de 2000 a agosto de 2001 na área experimental da Fazenda Vitorinha, situada no município de Lavras, região sul do Estado de Minas Gerais, localizada a $918 \mathrm{~m}$ de altitude, latitude $21^{\circ} 14^{\prime} \mathrm{S}$ e longitude $45^{\circ} 00^{\prime} \mathrm{W}$. A temperatura média anual do ar dessa região é de $19,4^{\circ} \mathrm{C}$ e as médias anuais de temperatura, máxima e mínima são de $26,1^{\circ} \mathrm{C}$ e $14,8^{\circ} \mathrm{C}$, respectivamente, com precipitação anual de 1529,7 mm (BRASIL, 1992). Segundo a classificação climática de Köppen, o clima da região é do tipo Cwa, apresentando duas estações definidas: seca, de abril a setembro, e chuvosa, de outubro a março.

Foram utilizados plantios consorciados de café (Coffea arabica L.) cv Rubi com seringueira (Hevea brasiliensis Muell Arg.) e plantios em monocultivo, implantados em 1997 e 1999, respectivamente. Foram avaliadas plantas de café e seringueira nos diferentes sistemas de cultivo (consórcio e monocultivo), constituindo os cinco tratamentos apresentados a seguir.

O primeiro tratamento refere ao consórcio em que as plantas de seringueira, clone PB235, foram plantadas como quebra-vento, margeando os talhões da lavoura cafeeira (consórcio tipo margem). No segundo tratamento, as plantas de seringueira, clone PB235, fo- ram plantadas em linhas duplas perpendiculares ao sentido das ruas de cafeeiros (consórcio tipo renque), sendo a distância entre os renques consecutivos de 20,0 m. Os demais tratamentos referem-se a plantios em monocultivo de cafeeiros e seringueiras, clones PB235 e GT1. As plantas de café avaliadas nos consórcios foram as situadas na linha vizinha à planta de seringueira avaliada.

Para as seringueiras plantadas em linha, como quebra-vento para as plantas de café, o espaçamento foi de 3,0 m entre plantas, sendo de $0,75 \mathrm{~m}$ a distancia entre a linha de seringueiras e a primeira rua de cafeeiros. No consórcio denominado tipo renque, o espaçamento foi de 4,0 x 3,0 m (entre plantas e entre linhas). Em relação ao monocultivo de seringueira, para ambos os clones avaliados, o espaçamento utilizado foi de 3,0 x 7 $\mathrm{m}$ (entre plantas e entre linhas). Tanto para o monocultivo como no consórcio o espaçamento entre as plantas de café era de 2,0 x 0,75 m.

Para avaliar o crescimento das seringueiras, foram realizadas medidas de diâmetro do caule e altura das plantas. A altura da planta foi considerada desde o ponto de enxertia até a região de inserção das folhas apicais, e o diâmetro do caule a $20 \mathrm{~cm}$ do solo. Essas avaliações foram realizadas em uma freqüência semanal para a altura das plantas, ao passo que o diâmetro foi medido em intervalos mensais ao longo de todo o período experimental.

Após sete meses de avaliação, foram realizadas coletas de 10 folhas em três plantas de cada espécie, em cada tratamento. Nas plantas de café, foram coletadas folhas completamente expandidas, situadas entre o quinto e oitavo nó, a contar do ápice de ramos ortotrópicos do terço superior da planta. Para as plantas de seringueira, foram retiradas folhas completamente expandidas de lançamentos anteriores ao último lançamento.

O material foi fixado em FAA (formaldeído, ácido acético e álcool etílico 70\%) por 72 horas, seguindo a metodologia de Johansen (1940), e posteriormente conservado em álcool $70 \%$, sendo o estudo anatômico baseado no exame microscópico de seções obtidas à mão livre. Essas foram clarificadas em solução com hipoclorito de sódio a $20 \%$ de produto comercial por um período que variou de três a cinco minutos; em seguida, foram lavadas em água destilada, neutralizadas em água acética $1 \%$ e montadas em glicerina a $50 \%$. O corante usado foi a mistura de azul de astrasafranina, seguindo-se os métodos descritos por Kraus e Arduin (1997). Para as avaliações relativas à caracteri- 
zação dos estômatos (número médio por $\mathrm{mm}^{2}$, diâmetro polar e equatorial), foram feitos cortes paradérmicos na região mediana das folhas na face abaxial e, como corante, empregou-se a safranina hidroalcoólica. A partir das seções transversais, foram efetuadas 20 medições, com o auxílio de ocular micrométrica de 5 plantas, das espessuras das epidermes superior (adaxial) e inferior (abaxial), dos parênquimas esponjoso e paliçádico, em um microscópio de campo claro (Carl Zeiss-Amplival). Em seguida, as lâminas foram observadas em microscópio Olympus CBB, segundo técnica de Labouriau et al. (1961). Na região mediana de cada folha, foram quantificados quatro campos, totalizando 40 campos por tratamento (dez folhas por tratamento), sendo 160 campos para a seringueira e 640 campos para o cafeeiro. As fotomicrografias foram obtidas em microscópio Olympus BX60 utilizando filme ASA 100.

O delineamento experimental utilizado foi o inteiramente casualizado (DIC), com parcelas subdivididas com quatro repetições por tratamento. A análise de variância foi feita utilizando-se do programa SISVAR e a comparação de médias foi realizada pelo teste Tukey ao nível de $5 \%$ de probabilidade.

\section{RESULTADOS E DISCUSSÃO}

Na Figura 1 verificam-se a evolução da altura e o diâmetro das plantas de seringueira ao longo do período experimental. Observa-se, como esperado, que a taxa de crescimento, independente do sistema de cultivo durante a estação chuvosa (outubro a março), foi maior que no período seco (abril a julho). Tal padrão de crescimento é similar ao verificado em plantios de seringueiras na Região Sudeste por Rocha Neto (1990) e Soares et al. (1993). As plantas do consórcio tipo renque apresentam maior altura do que aquelas dos outros sistemas de cultivo, e essas diferenças podem estar associadas à posição das plantas no terreno, que pode definir uma condição de maior exposição à radiação solar, menor exposição ao vento e ser mais favorável ao crescimento das plantas. Ainda existem as características das mudas, como capacidade de desenvolvimento do sistema radicular após transplantio, que podem também afetar a taxa de crescimento das plantas.

Ressalta-se que as menores taxas de crescimento observadas entre abril e junho são associadas à menor disponibilidade de água no solo e à queda da temperatura, característica desse período, que reduzem o metabolismo das plantas. Efeitos mais acentuados surgem quando a menor disponibilidade de radiação está associada a condições limitantes de oferta de água no solo (MENZEL e SIMPSON, 1994).

Com esses resultados, não se evidencia um padrão distinto de crescimento das plantas para os diferentes sistemas de cultivo, o que permite dizer que nessa fase de estabelecimento do consórcio a competição por luz, água e nutrientes, bem como as alterações do microclima esperadas nesses sistemas de cultivo (MONTEITH et al., 1991) ainda não são suficientes para alterar o padrão de crescimento das plantas.

Pelo estudo da superfície abaxial, em vista frontal, observa-se que em consórcio as seringueiras apresentaram um maior número de estômatos $/ \mathrm{mm}^{2}$, sendo o maior valor verificado para as plantas do consórcio margem (Tabela 1). Nota-se ainda um aumento do número de estômatos para o clone GT1 em relação ao PB235 nos tratamentos de monocultivo. Em relação à dimensão dos estômatos, os menores valores de diâmetros polar e equatorial foram encontrados no consórcio tipo renque (Tabela 1).

$\mathrm{O}$ aumento no número de estômatos $/ \mathrm{mm}^{2}$ geralmente é observado em folhas de plantas expostas a pleno sol, como relatado por vários autores, entre eles Knetcht e O’Leary (1972), Sílvia e Anderson (1985), Castro et al. (1998), Almeida (2001) e Zanela (2001), podendo indicar um mecanismo de adaptação das plantas às condições de baixa disponibilidade hídrica no solo. Essa característica, de acordo com Medri e Lleras (1980a), pode assegurar às plantas uma maior eficiência de trocas gasosas em horários caracterizados por maior umidade relativa do ar.

Foram observadas nas folhas de seringueira modificações na organização do parênquima clorofiliano dos clones avaliados nos diferentes sistemas de cultivo (Tabela 2 - Figura 2). Medri e Lleras (1980b) descreveram a morfologia interna da folha de seringueira em clones diplóides e poliplóides, na qual a lâmina foliar possui organização dorsiventral; entretanto, neste estudo, a lâmina dos clones estudados apresentaram organização isobilateral. Pela análise estatística da variação da espessura do limbo, do parênquima paliçádico e do parênquima lacunoso constatam-se diferenças significativas ao se comparar os sistemas de cultivo. 

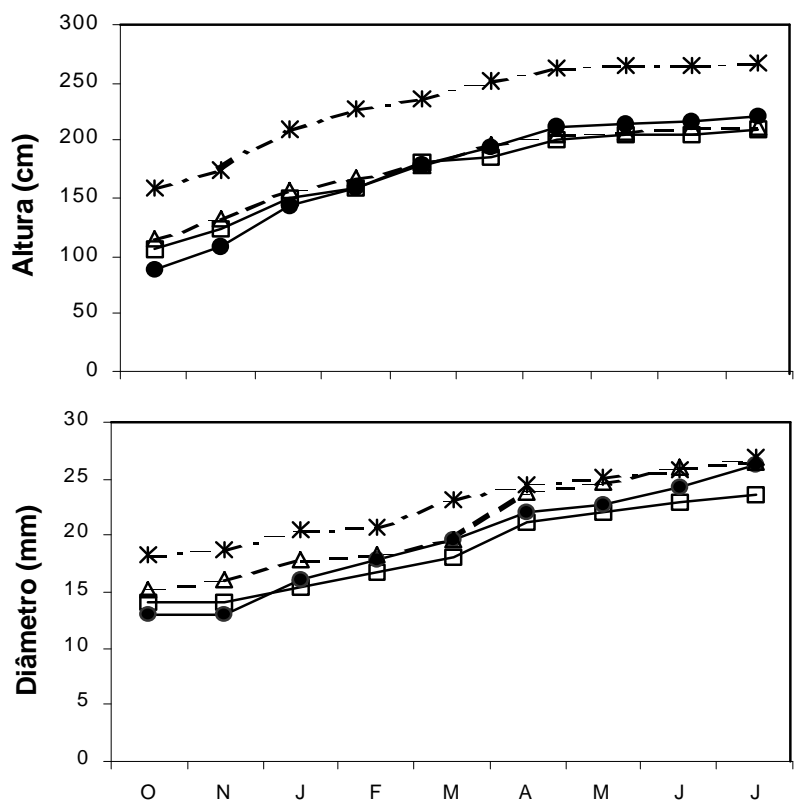

FIGURA 1 - Altura e diâmetro de plantas de seringueira em monocultivo clone GT1 (•), clone PB235 ( ) e em consorcio tipo margem ( ) e consórcio tipo renque (*). Cada ponto representa a média de 4 observações.

TABELA 1 - Número de estômatos por $\mathrm{mm}^{2}$ e diâmetros polar e equatorial dos estômatos de seringueira em monocultivo (M) e consórcio (C).

\begin{tabular}{|c|c|c|c|}
\hline Tratamento & $\mathbf{N}^{0}$ estômatos/mm² & Diâmetro polar $(\mu \mathrm{m})$ & Diâmetro equatorial $(\mu \mathrm{m})$ \\
\hline M-clone PB235 & $286,0 \mathrm{~d}$ & $25,94 a$ & $14,0 \mathrm{ab}$ \\
\hline M-clone GT1 & $348,17 b$ & $25,45 \mathrm{a}$ & $15,25 b$ \\
\hline $\mathrm{C}-$ renque & $336,33 \mathrm{c}$ & $23,27 b$ & $13,1 \mathrm{c}$ \\
\hline C- margem & $397,0 \mathrm{a}$ & $27,28 \mathrm{a}$ & $19,44 \mathrm{a}$ \\
\hline
\end{tabular}

Médias seguidas pela mesma letra não diferem entre si pelo teste de Tukey a $5 \%$ de probabilidade.

As células paliçádicas e do tecido lacunoso dos cafeeiros dos consórcios em margem e renque não diferiram significativamente daquelas do café em monocultivo (Tabela 3 - Figura 2). Como pode ser observado na Tabela 4, também não foram observadas diferenças significativas no número de estômatos $/ \mathrm{mm}^{2}$, no índice estomático, no diâmetro polar e no diâmetro equatorial.
Voltan et al. (1992), ao estudarem a variação da anatomia foliar de cafeeiros submetidos a diferentes intensidades luminosas, observaram diferenças entre as plantas cultivadas a pleno sol e em intensidades de luz intermediárias. Em diversos outros estudos é abordada a questão da intensidade de radiação solar no cafeeiro; entretanto, poucos trabalhos têm descrito a anatomia 
foliar dessas plantas crescendo em sistema de consórcio e monocultivo.

Pelos resultados aqui obtidos, verifica-se que, na fase de desenvolvimento dos sistemas de plantio estudados, existe pouca concorrência pelos fatores ambientais, indicando que as seringueiras não influenciaram as respostas anatômicas dos cafeeiros nessa fase do cul- tivo. Por outro lado, ressalta-se a necessidade da continuidade desses estudos para que se possa avaliar as interações entre as plantas e as possíveis alterações do microclima em diferentes fases do desenvolvimento nessas formas de cultivo, fundamentais para uma abordagem da questão do consórcio do cafeeiro com culturas perenes.

TABELA 2 - Espessura do limbo (ELi), parênquima palicádico (EPp), esponjoso(EPl), epiderme da superfície abaxial (FAb) e adaxial (FAd) de plantas de seringueira em monocultivo (M) e consorciados (C).

\begin{tabular}{cccccc}
\hline Tratamento & Eli $(\mu \mathbf{m})$ & $\mathbf{E P p}(\mu \mathbf{m})$ & EPI $(\mu \mathbf{m})$ & FAb $(\mu \mathbf{m})$ & FAd $(\mu \mathbf{m})$ \\
\hline M- clone P235 & $324,8 \mathrm{a}$ & $167,0 \mathrm{a}$ & $109,3 \mathrm{a}$ & $21,0 \mathrm{~b}$ & $27,7 \mathrm{a}$ \\
M - clone GT1 & $320,8 \mathrm{a}$ & $155,6 \mathrm{~b}$ & $111,8 \mathrm{a}$ & $23,3 \mathrm{a}$ & $30,1 \mathrm{a}$ \\
C- renque & $271,6 \mathrm{c}$ & $130,5 \mathrm{c}$ & $95,4 \mathrm{~b}$ & $19,7 \mathrm{~b}$ & $26,1 \mathrm{a}$ \\
S- margem & $293,7 \mathrm{~b}$ & $154,8 \mathrm{~b}$ & $91,3 \mathrm{~b}$ & $19,3 \mathrm{~b}$ & $28,3 \mathrm{a}$ \\
\hline
\end{tabular}

Médias seguidas pela mesma letra não diferem estatisticamente pelo teste de Tukey, ao nível de $5 \%$ de probabilidade.

TABELA 3 - Espessura do limbo (ELi), parênquima palicádico (EPp), esponjoso (Epl), epiderme da superfície abaxial (FAb) e adaxial (FAd) de plantas de cafeeiros em monocultivo e consorciados na região de Lavras- MG.

\begin{tabular}{cccccc}
\hline Tratamento & $\mathbf{E L i}(\boldsymbol{\mu} \mathbf{m})$ & $\mathbf{E P p}(\boldsymbol{\mu} \mathbf{m})$ & $\mathbf{E P I}(\boldsymbol{\mu} \mathbf{m})$ & $\mathbf{F A b}(\boldsymbol{\mu m})$ & FAd $(\boldsymbol{\mu} \mathbf{m})$ \\
\hline $\mathrm{C}$ & $590,2 \mathrm{a}$ & $143,2 \mathrm{~b}$ & $375,7 \mathrm{a}$ & $28,2 \mathrm{a}$ & $43,1 \mathrm{~b}$ \\
$\mathrm{Cr}$ & $593,3 \mathrm{a}$ & $150,2 \mathrm{a}$ & $367,0 \mathrm{a}$ & $30,0 \mathrm{a}$ & $46,1 \mathrm{a}$ \\
$\mathrm{Cm}$ & $607,2 \mathrm{a}$ & $153,8 \mathrm{a}$ & $376,1 \mathrm{a}$ & $29,7 \mathrm{a}$ & $48,1 \mathrm{a}$ \\
\hline
\end{tabular}

Médias seguidas pela mesma letra não diferem estatisticamente pelo teste de Tukey ao nível de 5\% de probabilidade.

TABELA 4 - Número de estômatos por $\mathrm{mm}^{2}$ (NE), índice estomático (IE) e diâmetros polar (DP) e equatorial (DE) de cafeeiros em monocultivo e consorciados.

\begin{tabular}{ccccc}
\hline Tratamento & NE & IE $(\%)$ & DP $(\mu \mathbf{m})$ & DE $(\mu \mathbf{m})$ \\
\hline $\mathrm{Cm}$ & $406,45 \mathrm{a}$ & $307,14 \mathrm{a}$ & $25,39 \mathrm{a}$ & $15,66 \mathrm{a}$ \\
$\mathrm{Cr}$ & $426,61 \mathrm{a}$ & $301,53 \mathrm{a}$ & $24,94 \mathrm{a}$ & $15,19 \mathrm{a}$ \\
$\mathrm{C}$ & $407,74 \mathrm{a}$ & $287,35 \mathrm{a}$ & $24,57 \mathrm{a}$ & $15,17 \mathrm{a}$ \\
\hline
\end{tabular}

Médias seguidas pela mesma letra não diferem entre si pelo teste de Tukey ao nível de $5 \%$ de probabilidade. 


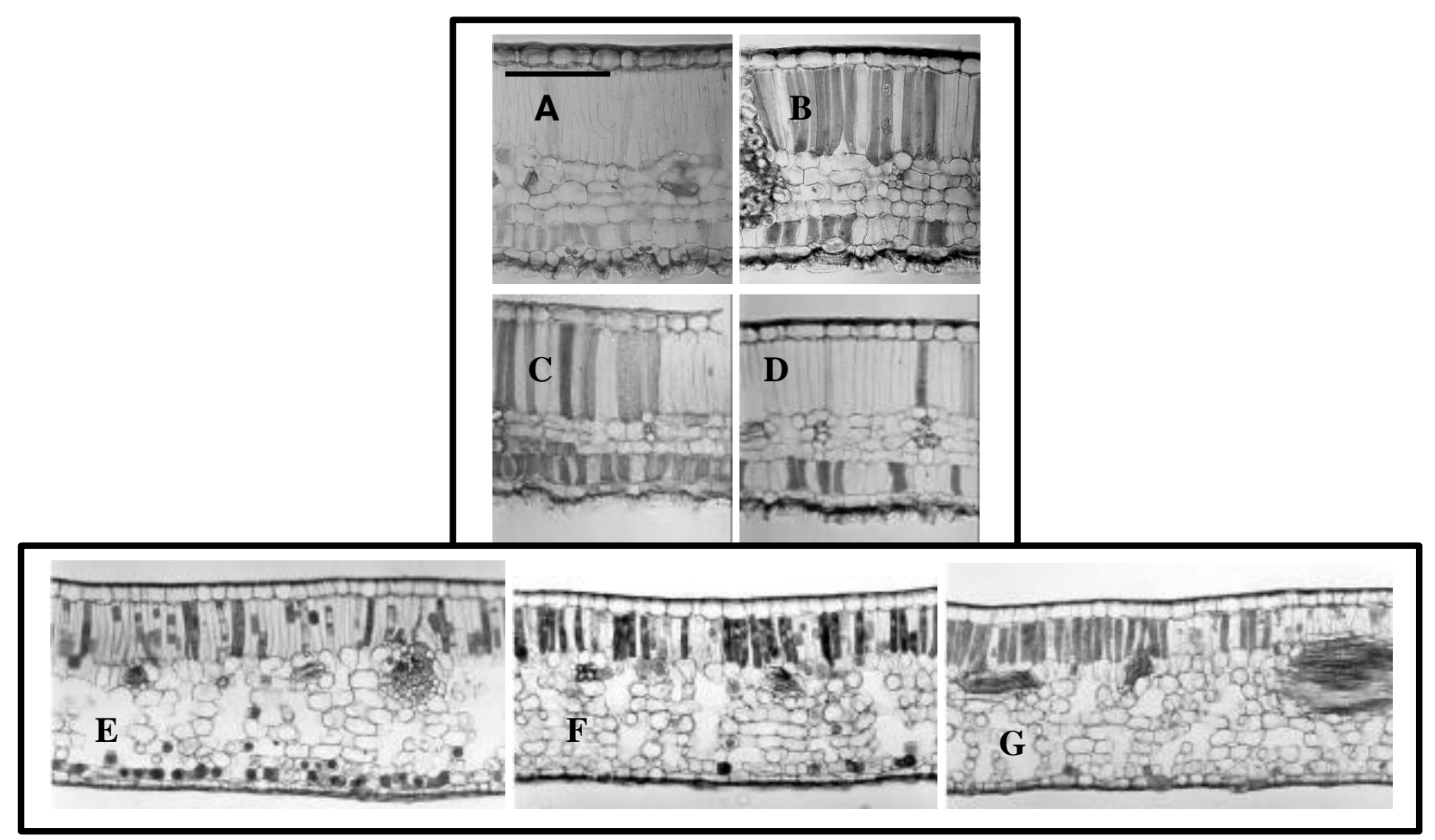

FIGURA 2 - Seção transversal de folhas de seringueiras em monocultivo A e B (GT1 e PB-235, respectivamente); do consórcio tipo margem $\mathbf{C}$ e do consórcio tipo renque D. E cafeeiros do consórcio tipo margem $\mathbf{E}$; no consórcio tipo renque F; e em monocultivo $\mathbf{G}$. A barra corresponde a $100 \mu \mathrm{m}$.

\section{CONCLUSÕES}

O sistema de cultivo, na fase de estabelecimento dos plantios, não influencia o padrão de crescimento da seringueira e anatomia foliar de cafeeiros.

Há alterações na anatomia foliar de seringueiras associadas ao sistema de cultivo e ao clone estudado.

\section{REFERÊNCIAS BIBLIOGRÁFICAS}

ABRAMS, M. D.; MOSTOLLER, S. A. Gas exchange, leaf structure and nitrogen in contrasting sucessional tree species growing in open and understory sites during a drought. Tree Physiology, Vicoria, v. 15, p. 361-370, 1995.

ALMEIDA, L. P. Germinação, crescimento inicial e anatomia foliar de plantas jovens de Cryptocarya aschersoniana Mez. sob diferentes níveis de radiação. 2001. 96 p. Dissertação (Mestrado em Fisiologia Vegetal) - Universidade Federal de Lavras, Lavras, 2001.
ASHTON, P. M. S.; BERLYN, G. P. Leaf adaptations of some Shorea species to sun and shade. New Phytologist, Cambridge, v. 121, p. 587-596, 1992.

BRASIL. Ministério da Agricultura e Reforma Agrária. Secretaria Nacional de Irrigação. Departamento Nacional de Meteorologia. Normais Climatológicas 19611990. Brasília, 1992. 84 p.

CASTRO, E. M. de; GAVILANES, M. L.; ALVARENGA, A. A. de; CASTRO, D. M. de; GAVILANES, T. O. T. Aspectos da anatomia foliar de mudas de Guarea guidonea (L.) Sleumer, sob diferentes níveis de sombreamento. Daphne, Belo Horizonte, v. 8 , n. 4 , p. $31-35,1998$.

DIJKMAN, M. J. Hevea: thirty years of research in Far East Florida. Miami: Universtiy of Miami, 1951. 87 p.

ESAU, K. Anatomia das plantas com sementes. São Paulo: EDUSP, 1974. 293 p. 
FAILS, B.S.; LEWIS, A. J.; BARDEN, J. A. Anatomy and morpholology of sun and shade-growth Ficus benjamina. Journal of the American Society for Horticultural Science, Alexandria, v. 107, n. 5, p. 754-757, 1982.

FANCELLI, A. L. Culturas intercalares e coberturas vegetais em seringais. In: SIMPÓSIO SOBRE A CULTURA DA SERINGUEIRA NO ESTADO DE SÃO PAULO, 1., 1986, Piracicaba. Anais... Campinas: Fundação Cargill, 1986. p. 229-243.

FANCELLI, A. L. Seringueira consorciada às culturas anuais perenes. In: SIMPÓSIO SOBRE A CULTURA DA SERINGUEIRA NO ESTADO DE SÃO PAUlO, 2., 1990, Piracicaba. Anais... Piracicaba: ESALQ, 1990. p. 205-222.

\section{INSTITUT DE RECHERCHES SUR LE CAOUTCHOUC. Rapport general 1990. Paris: IRCA/CIRAD, 1992. 219 p.}

KNETCHT, G. M.; O’LEARY, J. W. The efect of light intensity on stomatal density of Phaseolus vulgaris leaves. Botany, London, v. 133, p. 132-134, 1972.

KRAUS, J. E.; ARDUIN, M. Manual básico de métodos em morfologia vegetal. Rio de Janeiro: Seropédica, 1997. $198 \mathrm{p}$.

LABOURIAU, L. G.; OLIVEIRA, J. C.; SALGADOLABOURIAU, M. L. Transpiração de Schizolobiuem parahiba (Vell.). Toledo: comportamento na estação chuvosa, nas condições de Caeté, Minas Gerais, Brasil. Anais da Academia Brasileira de Ciências, Rio de Janeiro, v. 33, n. 2, p. 237-257, 1961.

LEE, D. W.; OBERBAUER, S. F.; JONSON, P.; KRISHNAPILAY, B.; MANSOR, M.; MOHAMAD, H.; YAP, S. H. Effects of irradiance and spectral quality on leaf structure and function in seedlings of two southeast Asian hopea (Dipterocarpaceae) spcies. American Journal of Botany, Columbus, v. 87, n. 4, p. $447-455,2000$.

MEDRI, E. M.; LLERAS, E. Aspectos da anatomia ecológica de folhas de Hevea brasiliensis Muell. Arg. Acta Amazônica, Manaus, v. 10, n. 3, p. 463-493, 1980a.

MEDRI, E. M.; LLERAS, E. Comparação anatômica entre folhas de um clone diploide (IAN 873) e dois clones poliploides (IAC 207, 222) de Hevea brasiliensis Muell. Arg. Acta Amazonica, Manaus, v. 11, n. 1, p. 35-47, 1980 b.
MENZEL, C. M.; SIMPSON, D. R. Passion- fruit. In: SCHAFFER, B.; ANDERSEN, P. C. (Eds.). Handbook of enviromental physiology of fruits crops. Boca Raton: CRC, 1994. v. 2, p. 225-241.

MONTEITH, J. L.; ONG, C. K.; CORLETT, J. E. Microclimatic interations in agroforestry systems. In: JARVIS, P. G. Agroforestry: principles and pratice. Amsterdan: Elsevier, 1991. 336 p.

PEETERS, L. Y. K.; SOTO-PINTO, L.; PERALES, H.; MONTOYA, G.; ISHIKI, M. Coffee production, timber, and firewood in traditional and Inga-shaded plantations in Southern Mexico. Agriculture, Ecosystems and Environment, [S.1.], v. 95, p. 481-493, 2003.

PEREIRA, J. da P. Seringueira: formação de mudas, manejo e perspectivas no Noroeste do Paraná. Londrina: IAPAR, 1992. 60 p. (Circular, 70).

PEREIRA, J. da P.; ANDROCIOLI FILHO, A.; LEAL, A. C.; RAMOS, A. L. M. Consorciação de seringueira e cafeeiro em fase terminal e o seu efeito na redução do período de imaturidade do seringal. In: CONGRESSO BRASILEIRO SOBRE SISTEMAS AGROFLORESTAIS, 1., 1994, Porto Velho. Anais... Colombo: EMBRAPA-CNPF, 1994. v. 1, p. 103-111. (Documentos, 27).

PEREIRA, A. V.; PEREIRA, E. B. C.; FIALHO, J. F. de; JUNQUEIRA, T. V. N.; MACEDO, R. L. G. Sistemas agroflorestais de seringueira com cafeeiro. Planaltina: EMBRAPA, 1998. 77 p. (Documentos, 70).

PEREIRA, J. P.; ANDROCIOLI FILHO, A.; LEAL, A. C.; RAMOS, A. L. M. Desenvolvimento vegetativo da seringueira em sistema agroflorestal com cafeeiro em fase terminal. IN: SIMPÓSIO INTERNACIONAL SOBRE ECOSSISTEMAS FLORESTIAS, 6., 2000, Porto Seguro. Anais... Rio de Janeiro: Biosfera, 2000. p. 4.

ROCHA NETO, O. G. da. Aspectos ecofisiológicos sazonais da produção de mudas de seringueira (Hevea spp), na região sudoeste do Brasil: efeito de estresses ambientais sobre o estado nutricional, o comportamento estomático e crescimento. 1990. 125 f. Tese (Doutorado) Universidade do Estado de São Paulo, Campinas, 1990.

SANTOS, A. J. dos; LEAL, A. C.; GRAÇA, L. R.; CARMO, A. P. C. do. Viabilidade econômica do sistema agroflorestal Grevílea x Café na região norte do Paraná. CERNE, [S.1.], v. 6, n. 1, p. 89-100, 2000. 
SCHALLER, M.; SCHROTH, G.; BEER, J.; JIMÉNEZ, F. Species and site characteristics that permit the association of fast-growing trees with crops: the case of Eucalyptus degulata as coffee shade in Costa Rica. Forest Ecology and Management, [S.1.], v. 175, p. 205-215, 2003.

SILVIA, E. A. M.; ANDERSON, C. E. Influência da luz no desenvolvimento foliar do feijoeiro (Phaseolus vulgaris L.). Revista Ceres, Viçosa, v. 32, n. 179, p. 1$11,1985$.

SOARES, A. M.; OLIVEIRA, L. E. M. O.; ROCHA NETO, O. G. da. Avaliação do sistema de produção de porta-enxertos de seringueira (Hevea brasiliensis Muell. Arg.) sob as condições edafoclimáticas de Lavras - Minas Gerais. Revista Árvore, Viçosa, v. 17, n. 2, p. 235 246, 1993.
VENEZIANO, W.; MEDRADO, M. J. S.; RIBEIRO, S. I.; LISBOA, S. de M.; MENEZES, L. C. C. de; COSTA, J. N. M.; SANTOS, J. C. F. Associação da seringueira com a cultura do cafeeiro no Estado de Rondônia. In: CONGRESSO BRASILEIRO SOBRE SISTEMAS AGROFLORESTAIS, 1., 1994, Porto Velho. Anais... Colombo: EMBRAPA-CNPF, 1994. v. 1, p. 121-133.

VOLTAN, R. B. Q.; FAHL, J. I.; CARRELI, C. C. Variação da anatomia foliar de cafeeiros submetidos a diferentes intensidades luminosas. Revista Brasileira de Fisiologia Vegetal, Londrina, v. 4, n. 2, p. 99-105, 1992.

ZANELA, S. M. Respostas ecofisiológicas e anatômicas ao sombreamento em plantas jovens de diferentes grupos ecológicos. 2001. 79 p. Dissertação (Mestrado em FisiologiaVegetal) - Universidade Federal de Lavras, Lavras, 2001. 